${ }^{8}$ Watring, W G, et al, fournal of Reproductive Medicine, 1976, 16, 35.

${ }^{9}$ Wesselius-de Casparis, A, et al, British Medical fournal, 1971, 3, 144

" Gibbens, G L D, and Chard, T, American fournal of Obstetrics and Gynecology, 1976, 126, 243.

${ }^{1}$ Ott, A, Hayes, J, and Polin, J, Obstetrics and Gynecology, 1976, 48, 362.

12 Lands, A M, et al, Nature, 1967, 214, 597.

13 Ingemarsson, I, American Fournal of Obstetrics and Gynecology, 1976, 125, 520.

14 Liggins, G C, and Vaughan, G S, fournal of ()bstetrics and Gynaecology of the British Commonicealth, 1973, 80, 29.

${ }^{15}$ Bieniarz, J, Ivankovich, A, and Scommegna, A, American forrnal of Obstetrics and Gynecology, 1974, 118, 910.

16 Ehrenkranz, R A, et al, American fournal of Obstetrics and Gynecology, 1976, 126, 343.

1: Brettes, J P, Renaud, R, and Gandar, R, American fournal of Obstetrics and Gynecology, 1976, 124, 164

'* Blouin, D, Murray, M A F, and Beard, R W, British fournal of ()bstetrics and Gynaecology, 1976, 83, 711 .

19 Nair, G V, Ghosh, A K, and Lewis, B V, Lancet, 1976, 1, 907.

2" British Medical fournal, 1976, 2, 1403.

21 Tamby Raja, R L, Anderson, A B M, and Turnbull, A C, British Medical fournal, 1974, 4, 67

\section{Pregnancy and oral contraceptives and the uterine vessels}

Few parts of the body show a dramatic change in the role of particular arteries once body growth has ceased. In female animals, however, the uterine and mammary arteries have to provide variable amounts of blood to match the remarkable changes in the size and function of uterus and breast throughout reproductive life. Arteries which need to be big and patent at certain times undergo striking changes when the flow requirements return to a basal level. Some of these changes are mediated by processes which we now regard as abnormal in more stable territories (thrombotic luminal occlusion, intimal hyperplasia, and medial calcification) but which serve to remodel the arterial tree to its new functions. Nevertheless, the way in which these changes are brought about may shed light on pathological thrombosis elsewhere and on atherosclerosis in general.

Gillman ${ }^{1}$ found that, regardless of the patients' age and previous parity, uterine arteries removed during 28 caesarean sections at term were all widely patent, though their walls contained large quantities of mucopolysaccharide. In contrast, of 55 sets of arteries removed at hysterectomy, 38 showed thrombi on their walls, 10 occlusive thrombi, and 18 appreciable medial calcification. The last correlated strongly with parity: it was, for example, particularly obvious in three women under 40 who had had seven or more children.

The animal whose arterial tree most closely resembles that of man is the pig, and in these animals Gillman showed that the degree of luminal, intimal, and medial change was directly related to parity. As the changes took some weeks to develop after birth, another pregnancy often overtook the shuttingdown process, when the arteries would be required to remodel yet again to provide the gravid uterus with its enhanced blood supply.

These comparative biological facts need to be borne in mind when we read of descriptions of "disease" in uterine arteries from women. Recently Osterholzer et $a l^{2}$ have reported the findings in a series of 60 hysterectomies in which the amount of intimal proliferation in uterine vessels correlated closely with the extent and duration of oral contraceptive exposure. Nevertheless, the indications for hysterectomy must have differed from those in Britain-six of the 60 patients were under 25 and 16 were under 30 . Moreover, though the paper indicated which women had a parity of three or more-vital in assessing whether the intimal proliferation was "biological" as described by Gillman" or "pathological" as implied by Osterholzer et $a l^{2}$-no information was given about the numbers of women with one or two pregnancies. There were, however, fewer multiparous patients in those with normal vessels than in those showing proliferation, which fits with the "biological" concept of parity leading to remodelling. Finally, if the uterine arteries were indeed diseased, then risk factors for arterial disease (other than the use of oral contraceptives) were not evenly distributed through the three histological groups: these factors including smoking and negro race, for example.

We need to know whether the arterial changes observed by Osterholzer $e t \mathrm{al}^{2}$ are indeed attributable to oral contraceptives and should therefore be considered as a disease of the uterine arteries, or whether they represent simple arterial remodelling which happened to coincide with steroid use, because of the age, parity, and existence of other risk factors in their subjects. Further studies are likely to be of value not only to workers on human arterial disease but also to anyone who is interested in the comparative anatomy of the blood supply to the uterine wall.

${ }^{1}$ Gillman, T, in Biological Aspects of Occlusive Vascular Disease, eds D G Chalmers and G A Gresham. London, Cambridge University Press, 1964 .

2 Osterholzer, H O, et al, Obstetrics and Gynecology, 1977, 49, 227.

\section{Choosing patients for chenodeoxycholic acid treatment}

Given the right patient, oral chenodeoxycholic acid will dissolve gallstones. ${ }^{1}$ Moreover, this treatment is both convenient and safe. But not all gallstones respond to chenodeoxycholic acid, and, since they may take some 12 months to dissolve, ${ }^{2}$ we need to know which patients will and which will not respond.

Radio-opaque gallstones are unsuitable for this form of treatment: there are sound theoretical reasons why these are not likely to dissolve, borne out by clinical experience. Next, since dissolution depends on the stone being bathed in bile that is unsaturated with cholesterol a response seems improbable when the gallbladder is radiologically nonfunctioning. Other factors to be considered before deciding on this treatment include the age, sex, and general health of the patient as well as the severity of the symptoms. Probably chenodeoxycholic acid does not alter the underlying metabolic defect which predisposes to saturated hepatic bile, so that treatment may well need to be lifelong. So it is likely to prove most valuable in older patients, and particularly in those with conditions that make surgery hazardous. Chenodeoxycholic acid is not recommended in women of childbearing age, and experts disagree whether it should be used in patients taking the contraceptive pill. The slow effect of the treatment makes it unsuitable in patients with severe biliary tract symptoms or jaundice. So, on the whole, experience with oral dissolution treatment for common bile duct stones has been disappointing. 
The ideal patient for chenodeoxycholic acid treatment is over 45 years old with a functioning gallbladder containing radiolucent gallstones. Even so, not all radiolucent stones respond, for the fact that they are radiolucent does not guarantee that they are rich in cholesterol and therefore likely to dissolve in bile that has been rendered unsaturated with cholesterol. The $x$-ray interpretation is likely to be most misleading when there are multiple small irregular stones, for in about one-fifth of patients these stones are composed of bile pigment. ${ }^{3}$ For this reason, an analysis of bile composition may help. Pigment stones occur in bile that is unsaturated with cholesterol, whereas for cholesterol stones the reverse is true. The association of radiolucent stones and saturated bile obtained by duodenal drainage is a reliable way of predicting that the gallstones are rich in cholesterol. ${ }^{4}$

With increasing experience we now know that the size of the gallstone is also an important determinant of outcome. Most small stones dissolve in between three and 12 months, whereas gallstones over $10 \mathrm{~mm}$ in diameter may need up to three years' treatment. ${ }^{25}$ Dissolution probably depends on the secretion of unsaturated hepatic bile, and most investigators have monitored biliary lipid composition in patients being treated with chenodeoxycholic acid. Patients likely to have their stones dissolved generally have bile that is unsaturated when judged by analysis of a random, fasting sample of bile-rich duodenal fluid. Conversion of saturation to unsaturation may be taken as an index of potential success, while failure to achieve unsaturation indicates that dissolution is unlikely and that the dose of chenodeoxycholic acid should be increased. ${ }^{5}$ Nevertheless, there are exceptions: stone dissolution has occurred in some patients in whom random bile samples have been found to be saturated. ${ }^{6} 7$ Obese patients appear to be resistant to chenodeoxycholic acid, and their bile remains saturated despite relatively large doses of the bile acid. ${ }^{2}$

Overall far fewer patients seem suitable for treatment with chenodeoxycholic acid than was thought at first; indeed, possibly only one in five of all patients with gallstones will prove to be candidates for medical treatment. So inevitably selection for suitability and prediction of success need a complex series of decisions and plan of surveillance. ${ }^{2}$

${ }^{1}$ Bouchier, I A D, British Medical fournal, 1976, 2, 870

2 Dowling, R H, Clinics in Gastroenterology, 1977, 6, 141

3 Bell, G D, et al, Gut, 1975, 16, 359.

4 Bruusgaard, A, et al, Scandinavian fournal of Gastroenterology, 1977, 12, 97.

5 Iser, J H, et al, New England fournal of Medicine, 1975, 293, 378

- James, O, Cullen, J, and Bouchier, I A D, Quarterly fournal of Medicine, $1975,44,349$.

7 Barbara, L, et al, Digestion, 1976, 14, 209.

\section{Planning treatment for rheumatoid arthritis}

There is no treatment for severe rheumatoid arthritis that is both safe and really effective. Analgesic and anti-inflammatory drugs such as aspirin produce partial symptomatic relief at a high cost in toxicity. Corticosteroids and corticotrophin are the only agents which predictably suppress the inflammatory process, but harmful side effects preclude their use in all but extreme cases. The slow-acting drugs such as gold produce a modest but measurable effect in the longer term but expose the patient to hazards such as bone marrow aplasia.

Against this background the pharmaceutical industry has sought to produce new nonsteroidal anti-inflammatory drugs without the toxicity of aspirin, particularly gastric irritation. Numerous preparations have appeared for which these claims are made, usually heralded by sales promotion on a scale that leaves no doubt about the profits to be made from a product which enjoys even temporary popularity. Many of these drugs are propionic-acid derivatives, which tend to be both somewhat less potent and less toxic than aspirin in full doses. Opinions differ whether these preparations have replaced or should replace aspirin as the first line of treatment in rheumatoid arthritis; but there does seem to have been a steady shift in prescribing habit away from aspirin and towards the newer drugs. This may be more obvious in countries such as Britain, where the consumer is cushioned against the high cost of these preparations.

What, then, should be the policy in prescribing for patients with rheumatoid arthritis? The apparent confusion of choices may be simplified by a classification such as that put forward by Huskisson, ${ }^{1}$ who grouped the drugs into five categories. Firstly, the simple analgesics: paracetamol, codeine, Distalgesic (paracetamol and dextropropoxyphene), and aspirin in small doses ( $2 \mathrm{~g}$ daily or less). Group two includes the analgesics with minor anti-inflammatory properties: ibuprofen, naproxen, ketoprofen, mefenamic acid; group three those with major anti-inflammatory properties: indomethacin, phenylbutazone and aspirin in full doses (at least $3.6 \mathrm{~g}$ daily). Fourth are the pure anti-inflammatory drugs, corticosteroids and corticotrophin, and fifth the "slow acting" drugs: gold, penicillamine, the antimalarials, and immunosuppressives.

Those who believe that aspirin is no longer the sheet anchor of treatment should start the new patient on a drug from group two. If pain is not relieved, this may be supplemented with single doses of a group one drug. Patients with morning stiffness benefit from indomethacin, up to $100 \mathrm{mg}$ on retiring (capsules or suppository). If symptoms are still not relieved then group three drugs should be given, starting with full doses of aspirin and supplementing with a group one drug. If the disease remains active and progressive joint destruction occurs, use of a slow-acting drug should be considered. Corticosteroids should be reserved for patients with serious systemic disease (vasculitis) or for those in whom unacceptable pain and disability due to inflammation cannot be controlled by other means.

This scheme relies on non-steroid anti-inflammatory analgesics. Which of these newer preparations should be chosen? Huskisson $e t a l^{2}$ compared ibuprofen, fenoprofen, naproxen, and ketoproten in a short double-blind crossover trial. Naproxen combined greater effectiveness with a lower incidence of side effects and emerged as first choice; but the differences between the drugs were not great, and there was considerable individual variation, some patients doing well on one preparation, others on another. These drugs should, therefore, be seen as alternatives. In a more extended trial Mowat et $\mathrm{al}^{3}$ showed that naproxen $(250 \mathrm{mg}$ twice daily) continued to give satisfactory results over 10 months of treatment.

In conclusion it is sadly necessary to point out how seldom the individual patient benefits from drug treatment to the extent implied in many drug advertisements-or indeed in many of the enthusiastic drug trial reports. And all these drugs are potentially toxic; even naproxen, the current front runner, may occasionally cause severe gastrointestinal haemorrhage. ${ }^{4}$

\footnotetext{
${ }^{1}$ Huskisson, E C, Reports on Rheumatic Diseases, no 54. London, Arthritis and Rheumatism Council, 1974.

${ }^{2}$ Huskisson, E C, et al, British Medical fournal, 1976, 1, 1048.

${ }^{3}$ Mowat, A G, et al, Annals of the Rheumatic Diseases, 1976, 35, 498.

4 Hart, F D, and Matts, S G F, British Medical Fournal, 1974, 2, 51.
} 